\title{
Muscle function and omega-3 fatty acids in the prediction of lean body mass after breast cancer treatment
}

\author{
Cameron McDonald*, Judy Bauer, Sandra Capra and Mary Waterhouse
}

\begin{abstract}
Background: Decreased lean body mass (LBM) is common in breast cancer survivors yet currently there is a lack of information regarding the determinants of LBM after treatment, in particular, the effect of physical activity and dietary factors, such as long-chain omega-3 fatty acids (LCn-3) on LBM and LBM function. This cross-sectional study explored associations of LBM and function with LCn-3 intake, dietary intake, inflammation, quality of life (QOL) and physical fitness in breast cancer survivors to improve clinical considerations when addressing body composition change.
\end{abstract}

Methods: Forty-nine women who had completed treatment (surgery, radiation and/or chemotherapy) were assessed for body composition (BODPOD), LCn-3 content of erythrocytes, C-reactive protein (CRP), QOL, dietary intake, objective physical activity, 1-min push-ups, 1-min sit-stand, sub-maximal treadmill (TM) test, and handgrip strength.

Results: After adjustment for age, LBM was associated with push-ups $(r=0.343, p=0.000)$, stage reached on treadmill (StageTM) $(r=0.302,0.001)$, \% time spent $\geq$ moderate activity (Mod $+V i g)(r=0.228, p=0.024)$. No associations were seen between anthropometric values and any treatment, diagnostic and demographical variables. Body mass, push-ups and StageTM accounted for $76.4 \%$ of the variability in LBM (adjusted $r$-square: $0.764, p=0.000$ ). After adjustment docosahexanoic acid (DHA) was positively associated with push-ups ( $\beta=0.399, p=0.001$ ), eicosapentanoic acid (EPA) was negatively associated with squats $(r=-0.268, p=0.041)$, with no other significant interactions found between LCn-3 and physical activity for LBM or LBM function.

Conclusion: This is the first investigation to report that a higher weight adjusted LBM is associated with higher estimated aerobic fitness and ability to perform push-ups in breast cancer survivors. Potential LCn-3 and physical activity interactions on LBM require further exploration.

Keywords: Breast cancer; Omega-3 fatty acids; Lean body mass; Fitness; Nutrition; Exercise

\section{Introduction}

Loss of lean body mass (LBM) and simultaneous gains in fat mass are amongst the most common side effects following treatment for breast cancer (Mcdonald et al. 2011). This pattern of body composition change is distressing for the survivors and it is related to higher levels of chronic inflammation (Mourtzakis \& Bedbrook 2009), and a greater risk for metabolic syndrome (Healy et al. 2010) and its related diseases (Healy et al. 2010; Pierce et al. 2009). A growing literature has established LBM, and in particular skeletal muscle tissue, as an influential organ in hormonal, immune and metabolic function (Pedersen \& Febbraio 2012). Lifestyle factors such as physical activity and nutrient intake can enhance LBM size (Irwin et al. 2009) and function, (Courneya et al. 2007; Schmitz et al. 2005) and have also been associated with improved survival (Ibrahim \& Al-Homaidh 2010) and quality of life (Mcneely et al. 2006) after treatment for breast cancer. Taken together, LBM is becoming an important marker for women who have been diagnosed with breast cancer.

\footnotetext{
* Correspondence: c.mcdonald4@uq.edu.au

University of Queensland, Brisbane 4059, Australia
} 
Findings from observational studies have indicated that chemotherapy has been associated with declines of LBM during and after treatment (Cheney et al. 1994; Demark-Wahnefried et al. 1997; Demark-Wahnefried et al. 2001; Gordon et al. 2011; Kutynec et al. 1999), however not all trials have reported LBM loss after chemotherapy (Campbell et al. 2007). In contrast, associations between higher LBM and aromatase inhibitor hormonal therapy have been reported in three different data sets (Francini et al. 2006; Montagnani et al. 2008; Van Londen et al. 2011). Modifiable variables such as dietary intake and physical activity have not been extensively explored with regard to LBM change in breast cancer populations. Some evidence exists for an association between decreased physical activity and increased adiposity (Irwin et al. 2005), while mixed results have been reported in relation to dietary intake and adiposity, (Sheean et al. 2012) however a deeper understanding of physical activity, dietary factors and LBM change are needed to better guide clinicians in the post-treatment period.

Long chain omega-3 fatty acids (LCn-3) are established as anti-inflammatory agents and have been shown to protect LBM in cancer populations (Dewey et al. 2001; Murphy et al. 2012; Ries et al. 2012; Van Der Meij et al. 2011). However, conclusions from reviews of intervention studies in cancer populations investigating the effect of LCn-3's on LBM have been mixed (Murphy et al. 2012; Ries et al. 2012). Typically, older studies have shown a protective effect for LBM when the appropriate dose of LCn-3 is consumed (Fearon et al. 2006; Fearon et al. 2003). More recent studies investigating $2 \mathrm{~g}$ of EPA LCn-3 supplementation in individuals undergoing chemotherapy for non-small cell lung cancer (NSCLC) have shown significantly greater attenuation of LBM and improved levels of intra-muscular triglyceride (IMTG), compared to those not supplementing. (Murphy et al. 2010; Murphy et al. 2011). In non-cancer populations the effect of LCn-3 on LBM has been minimal, with the majority of controlled trials indicating limited clinical effect (Mcdonald et al. 2013b).

Recent research has indicated that a greater effect may be seen when LCn-3 s are combined with an anabolic stimulus (Mcdonald et al. 2013b; Rodacki et al. 2012; Smith et al. 2011a; Smith et al. 2011b). Three small, well controlled studies combined LCn-3 supplementation with exposure to an anabolic stimulus, i.e. hyperinsulinaemic/hyperaminoacidaemic clamp or resistance training. Two reported an increased muscle protein synthetic (MPS) response to for young healthy (Smith et al. 2011b), and elderly participants (Smith et al. 2011a), yet LCn-3 alone made no difference to basal MPS. The third study that used resistance training reported increased peak torque development for the supplemented group above that of the group who received the resistance training program only (Rodacki et al. 2012). Considering LBM function, measured by strength or power development, may be more important to health outcomes than absolute values of LBM, (Newman et al. 2006; Ruiz et al. 2008) further investigations are required.

Therefore, the objectives of this cross-sectional study was to explore associations of LBM and LBM function in the context of LCn-3 intake, dietary energy and protein intake, inflammation, quality of life (QOL) and parameters of physical fitness and activity in women who had completed breast cancer treatment. A secondary goal was to determine the effect of interactions between tissue content of LCn-3 and markers of physical fitness on LBM after treatment for breast cancer.

\section{Methods}

\section{Study design}

All participants provided written informed consent. The data presented here was collected as the baseline assessment for a 6-month 3-arm randomized controlled trial (RCT) investigating LBM in women who have completed treatment for breast cancer. Detailed rationale study protocol for the full trial has been published previously (Mcdonald et al. 2013a). The study was approved by the Uniting Care (UCH HREC: \#1034) and the University of Queensland (\#2011000079).

\section{Participants}

Participants were invited to participate through hospital breast cancer oncology centres, radio advertising, social media and breast cancer research registries in Brisbane, Australia. Baseline assessment occurred over one week, which included two visits 7 days apart.

\section{Eligibility}

Women $\geq 18$ years of age; had been diagnosed with early stage breast cancer (Stage 0-IIIa as determined by the American Joint Committee on Cancer Care); had successfully completed surgery, radiation and/or chemotherapy in the last 12 months (participants could be currently receiving endocrine and/or herceptin therapy); were able to perform moderate intensity physical activity, and have a BMI of $>20$ and $<35 \mathrm{~kg} / \mathrm{m}^{2}$ were eligible for enrolment. Participants were excluded if they had presence of metastatic growth or local/distal recurrence of cancer; a diagnosis of cardiovascular disease or diabetes; or, consumed $>1$ g of eicosapentanoic acid (EPA) and docosahexanoic acid (DHA) LCn-3 s combined per day.

\section{Measures}

\section{Anthropometric variables}

Height was measured to the nearest $0.5 \mathrm{~cm}$ using a stadiometer (Seca). Weight to the nearest $0.1 \mathrm{~kg}, \mathrm{LBM}$ and 
fat mass were measured using the BODPOD digital scales and air displacement plethysmography (ADP) pod (BODPOD, COSMED USA Inc), respectively. Before each assessment day, the BODPOD scales and air chamber were calibrated as per the manufacturer's instructions using known weights and volumes. All measures were performed by a certified BODPOD assessor. Results were expressed as percentage LBM and body fat of total weight, then absolute LBM was calculated giving a value in kilograms of LBM.

\section{Quality of Life (QOL)}

QOL was measured using the Functional Assessment of Cancer Therapy- Breast + 4 (FACT-B + 4) tool (Cella et al. 1993). That FACT-F subset of questions was also added to capture participant fatigue. Higher scores are representative of better well-being.

\section{Diet history}

Dietary intake was measured by the practitioner assisted Diet History Questionnaire (Martin 2004). Participants were asked to complete the questionnaire based on their intake over the last month. An accredited practicing dietitian reviewed the questionnaire with the participant to clarify portion sizes and other relevant details. Nutrient analysis was carried out using Foodworks 7 (Xyris Software).

\section{Blood analyses}

Fasting high sensitivity-C Reactive Protein (CRP) was measured using a latex-enhanced immunoturbidimetric assay of blood serum. The $8.5 \mathrm{ml}$ sample of whole blood was collected and analysed for CRP, then frozen at $-20^{\circ} \mathrm{C}$ for transport to Victoria, Australia for fatty acid testing.

Lipids from red cells were extracted with chloroform methanol mixture. The fatty acids were trans-esterificated to methyl esters with methylation reagent "Meth-Prep 2". The methylation extract was then analysed by gas liquid chromatography method with flame ionisation detection (gas chromatograph Schimadzu G-2010-FID). The proportion of fatty acids content of the erythrocytes expressed as $\%$ of total fatty acids.

\section{Muscle function and fitness tests}

Grip strength was performed on both arms, with the maximum of 3 attempts recorded. Participants were seated with feet flat on floor, shoulder in neutral position with elbow bent at 90 degrees. Upper body muscular strength-endurance was measured using a 1-minute push-up test. Participants were asked to perform as many push-ups (knees on ground) as possible in $1 \mathrm{mi}-$ nute (American College of Sports Medicine 2010).

Lower body muscular endurance was measured using a 1-minute sit-stand test. The participant was asked to perform as many sit-stand movements as possible in 1 minute. Chair height was standardised at $43 \mathrm{~cm}$ height (American College of Sports Medicine 2010).

Sub-maximal aerobic capacity was measured using the modified Balke sub-maximal treadmill test. Seated blood pressure was measured before each assessment to ensure safety of exercise (Sharman \& Stowasserb 2009). The test being completed when the individual had reached $85 \%$ of their estimated maximum heart rate ( $\max \mathrm{HR}$ ) (est. $\operatorname{maxHR}=220$-age).

\section{Statistical analysis}

Baseline characteristics were compared between treatment types and stages of disease using independent samples t tests or ANOVA. Spearman's correlation coefficient was used assess the strength of bivariate associations, \% time in moderate and vigorous activity were grouped together into one variable: $\%$ time in $\geq$ moderate activity. To assess the significance of age- and/or weight-adjusted associations between an outcome and a potential predictor, multivariable linear regression was used. Multivariable linear regression was used to model LBM as a function of various markers of fitness while also controlling for total body mass. For missing data, only those with full data sets were included in the models. The variables considered for inclusion in the model were those that were individually associated with LBM after adjusting for age and weight. Markers of fitness were added to the model sequentially, with the order determined by decreasing $r$-values. A predictor was only retained in the model if its coefficient was significantly different from zero at the 0.05 level. Adjusted R-squared was used to compare nested models. Models were also fitted that included interaction terms that explored the respective LCn-3 indices combined with fitness markers on LBM.

\section{Results}

Participants were recruited over a 15-month period (Oct 2011 - Jan 2013). A total of 135 women were initially screened for inclusion criteria. The major reasons for exclusion were $>12$ months post treatment completion and daily consumption of $>1000 \mathrm{mg}$ EPA and DHA combined. Forty-nine participants were eligible for the study and completed baseline assessment. Descriptive statistics of the population are shown in Table 1.

\section{Age}

Age was positively correlated with improved breast cancer related QOL $(\mathrm{r}=0.379, \mathrm{p}=0.007)$, fatigue $(\mathrm{r}=0.311$, $\mathrm{p}=0.30)$ and EPA $(\mathrm{r}=0.339, \mathrm{p}=0.026)$, and negatively correlated with $\%$ of time in vigorous activity $(r=-0.342$, $\mathrm{p}=0.022)$ and number of squats performed in 1-minute $(\mathrm{r}=-0.363, \mathrm{p}=0.011)$. 
Table 1 Characteristics of participants

\begin{tabular}{|c|c|}
\hline Characteristic $(n=49)$ & Value \\
\hline Age in years (mean; SD) & $48.6 \pm 9.5$ \\
\hline \multicolumn{2}{|l|}{ Race $(n, \%)$} \\
\hline —Caucasian & $44(88)$ \\
\hline —Asian & $3(6)$ \\
\hline -African & $1(2)$ \\
\hline —Asian Pacific Islander & $1(2)$ \\
\hline Anthropometric & (mean; SD) \\
\hline Height (m) & $1.65 \pm 0.07$ \\
\hline BMI (kgm-2) & $26.6 \pm 4$ \\
\hline Body mass (kg) & $73.1 \pm 13.8$ \\
\hline LBM (kg) & $43.6 \pm 5.6$ \\
\hline Body fat $\%$ & $39.5 \pm 6.9$ \\
\hline Waist (cm) & $85.4 \pm 11.1$ \\
\hline Hip Girth (cm) & $106.3 \pm 9.2$ \\
\hline $\mathrm{CRP}(\mathrm{n}=45 ; \text { med; range })^{*}$ & $(0.1-10.1)$ \\
\hline Total \% RBC n-3 $(n=43)^{*}$ & $5.9 \pm 1.6$ \\
\hline$\%$ EPA & $1.1 \pm 0.5$ \\
\hline$\% \mathrm{DHA}$ & $2.9 \pm 0.9$ \\
\hline \multicolumn{2}{|c|}{ Charaterstic of Disease (n; \%) } \\
\hline $0-1$ & $13(26)$ \\
\hline Ila & $19(28)$ \\
\hline \|b--IIla & $17(34)$ \\
\hline Estrogen receptor + ve & $39(78)$ \\
\hline HER-2 receptor + ve & $12(24)$ \\
\hline \multicolumn{2}{|l|}{ Treatment variables $(\mathrm{n} ; \%)$} \\
\hline Had Chemotherapy & $41(92)$ \\
\hline Taxane - Yes & $37(74)$ \\
\hline \multicolumn{2}{|l|}{ Radiation therapy } \\
\hline Tamoxifen & $13(26)$ \\
\hline $\mathrm{Al}$ & $20(40)$ \\
\hline None & $16(32)$ \\
\hline Time since completion $R x$ & $165 \pm 107$ \\
\hline
\end{tabular}

Al-Aromatase inhibitors.

*Missing data.

Associations of diagnostic and treatment related variables Compared to those diagnosed with earlier stage disease (0-IIa), those with later stage disease (IIb-IIIa) reported poorer results for BrCa related QOL (89.2 \pm 9.3 vs $79.3 \pm 15.7 ; \mathrm{p}=0.009)$, fatigue $(130.5 \pm 15.3$ vs $113.3 \pm$ 24.7; $\mathrm{p}=0.006$ ), total score for Greene climacteric scale $(11.8 \pm 6.8$ vs. $17.5 \pm 10.2 ; \mathrm{F}=5.308, \mathrm{p}=0.026)$, with specifically worse symptom scores reported for psychological, anxiety, depression and somatic fields (all $\mathrm{p}<0.05)$. Stage of disease was not associated with any indices of body composition, LCn-3 or physical function.
Compared to those who did not have radiation therapy, DHA values $(\mathrm{t}=2.904 ; \mathrm{p}=0.016)$ and $\mathrm{LCn}-3$ : $\mathrm{LCn}-6$ $(t=3.06 ; p=0.004)$ ratios were higher for those who underwent radiation therapy. Otherwise, radiation therapy was not associated with markers of body composition, QOL, dietary intake, LBM function, endurance or physical activity. Individuals taking tamoxifen tended to have lower EPA content compared to those taking AIs or no hormonal therapy $(0.78 \%$ vs. $1.16 \%$ \& $1.23 \%$; $\mathrm{F}=$ $3.153, p=0.054)$, however, there was no evidence to support an association between hormonal treatment and other markers of body composition, QOL, dietary intake, LBM function or physical activity.

\section{Associations between LBM and dietary intake, inflammation, physical activity, markers of fitness and quality of life}

LBM was positively correlated with daily intake of total energy $(\mathrm{r}=0.301, \mathrm{p}=0.036)$ and protein $(\mathrm{r}=0.464, \mathrm{p}=$ 0.001 ), and negatively correlated with higher squat test results $(\mathrm{r}=-0.39, \mathrm{p}=0.006)$ (Table 2). However, after adjusting for weight and age, the only significant associations with LBM were $\%$ time spent in $\geq$ moderate intensity activity ( $(3: 0.228, \mathrm{p}=0.024)$, number of push-ups performed ( $(3: 0.343, \mathrm{p}=0.000$ ) and treadmill stage completed ( $(: 0.302, p=0.001$ ) (Table 2 ). CRP was positively correlated with body fat \%, waist and hip however, these associations were no longer significant after controlling for total body weight (data not shown).

Associations between LCn-3 and anthropometric indices, inflammation \& quality of life after breast cancer treatment

No significant correlations were identified between absolute LBM or \% LBM for total RBC n-3, ratio of AA: EPA or \% RBC content of EPA or DHA (Table 3). No significant relationships were found between any other anthropometric variables and $n-3$ related values.

No significant correlations were identified between CRP and erythrocyte LCn-3. No markers of body composition, CRP or indices of LCn-3 intake were significantly correlated with either measure of QOL.

\section{Predictors of LBM in women soon after breast cancer}

Number of push-ups, StageTM, and mod + vig activity were considered for inclusion in a weight-adjusted linear regression model for LBM (Table 2). Table 4 shows coefficients for the variables included in the final model. Table 4 also shows the value of adjusted R-squared obtained as each variable was successively added to the model. Mod + vig was not retained in the final model because the coefficient was not significantly different from zero $(\beta=0.115, p=0.177)$ in the presence of the other predictors. The model including weight, push ups and 
Table 2 Associations between markers of absolute LBM and markers of LCn-3 intake, dietary intake, physical activity and fitness adjusted for weight $\&$ age $n$ value

\begin{tabular}{|c|c|c|c|c|c|c|c|c|}
\hline & \multicolumn{4}{|c|}{ Lean body mass (kg) } & \multicolumn{4}{|c|}{ Body fat $\%$} \\
\hline & \multicolumn{2}{|c|}{ Unadjusted } & \multicolumn{2}{|l|}{ Adjusted } & \multicolumn{2}{|c|}{ Unadjusted } & \multicolumn{2}{|l|}{ Adjusted } \\
\hline & $R$ & $p$-value & Standardise d B-coefficient & $p$-value & $\mathrm{R}$ & $p$-value & Standardise d B-coefficient & p-value \\
\hline Total daily kJ intake & 0.301 & 0.036 & 0.135 & 0.132 & -0.82 & 0.576 & -0.192 & 0.065 \\
\hline \multicolumn{9}{|l|}{ Total daily protein } \\
\hline intake & 0.464 & 0.001 & 0.144 & 0.121 & 0.04 & 0.787 & -0.197 & 0.068 \\
\hline CRP* & 0.258 & 0.083 & -0.128 & 0.238 & 0.597 & 0 & 0.123 & 0.343 \\
\hline \multicolumn{9}{|l|}{$\%$ time in sedentary } \\
\hline activity* & 0.167 & 0.273 & 0.053 & 0.579 & 0.23 & 0.88 & -0.058 & -0.524 \\
\hline$\%$ time in light activity* & -0.149 & 0.329 & -0.104 & 0.272 & 0.156 & 0.305 & 0.125 & 0.258 \\
\hline \multicolumn{9}{|l|}{$\%$ time in $>$ moderate } \\
\hline activity* & -0.041 & 0.787 & 0.228 & 0.024 & -0.466 & 0.001 & -0.275 & 0.015 \\
\hline Push up (in 1-min)* & -0.045 & 0.760 & 0.343 & 0.000 & -0.671 & 0 & -0.457 & 0 \\
\hline Squats (in 1-min)* & -0.39 & 0.006 & 0.044 & 0.71 & -0.454 & 0.001 & -0.098 & 0.439 \\
\hline \multicolumn{9}{|l|}{ Stage of treadmill } \\
\hline completed & -0.047 & 0.746 & 0.302 & 0.001 & -0.575 & 0 & -0.39 & 0 \\
\hline FACT $-B+4$ & -0.13 & 0.373 & 0.038 & 0.699 & -0.146 & 0.316 & -0.009 & 0.936 \\
\hline FACT-F & -0.128 & 0.381 & 0.023 & 0.813 & -0.133 & 0.362 & -0.002 & 0.982 \\
\hline
\end{tabular}

$\%$ time in activity: Accelerometry; \% time in > moderate activity: moderate and vigorous activity grouped together; Stage of treadmill completed: at which $85 \%$ of estimated HRmax was reached; *Reduced data: CRP: $n=46$; Accelerometer data: $n=45$; Push-ups: $n=48 ;$ Squats: $n=48$.

StageTM explained $76.4 \%$ of the variation in absolute LBM (Table 4).

Interactions of physical activity and indices of LCn-3 intake on markers of LBM function

The number of push-ups performed was positively correlated with time spent in $\geq$ moderate intensity activity $(\mathrm{r}=0.467 ; \mathrm{p}=0.001)$, total $\mathrm{n}-3$ levels $(\mathrm{r}=0.385 ; \mathrm{p}=$ $0.012)$ and DHA levels $(\mathrm{r}=0.517, \mathrm{p}=0.000)$ (Table 5). The correlation with total $n-3$ levels was no longer statistically significant after adjusting for DHA. DHA maintained a significant association after adjusting for age, weight, LBM and $\%$ time $>\bmod$ activity $(\beta=0.399, p=$ $0.001) \% \geq$. Mod activity remained a significant predictor (F-Test: $8.95, \mathrm{p}=0.005$ ) of the number of push-ups performed in one minute after adjusting for DHA, age, weight and LBM. There were no significant interactions between RBC LCn-3 and time spent in any intensity of activity for any of the regression models of physical function (data not shown).

\section{Discussion}

This paper reports a positive relationship between LBM (adjusted for total weight) and physical function represented by

Table 3 Univariate associations between indices of erththrocyte LCn-3 s and markers of body composition, inflammation and quality of life $(n=43)$

\begin{tabular}{lcccccccc}
\hline & \multicolumn{2}{c}{ Total $\mathbf{n}$-3 } & \multicolumn{2}{c}{ EPA } & \multicolumn{2}{c}{ DHA } & \multicolumn{2}{c}{ AA/EPA } \\
\hline & $r$ & $p$-value & $r$ & $p$-value & $r$ & $p$-value & $r$ & $p$-value \\
Weight $(\mathrm{kg})$ & 0.083 & 0.595 & 0.249 & 0.107 & -0.088 & 0.576 & -0.232 & 0.134 \\
LBM $(\mathrm{kg})$ & 0.093 & 0.554 & 0.156 & 0.319 & -0.27 & 0.864 & -0.159 & 0.309 \\
Body fat \% & 0.037 & 0.816 & 0.222 & 0.153 & -0.107 & 0.493 & -0.181 & 0.245 \\
Waist (cm) & 0.123 & 0.431 & 0.280 & 0.069 & -0.061 & 0.697 & -0.167 & 0.284 \\
Hip (cm) & 0.055 & 0.728 & 0.280 & 0.069 & -0.141 & 0.366 & -0.313 & $\mathbf{0 . 0 4 1}$ \\
CRP (mmol/L) & 0.035 & 0.822 & 0.183 & 0.241 & -0.42 & 0.791 & -0.224 & 0.149 \\
FACT-B + 4 & -0.063 & 0.689 & 0.039 & 0.804 & -0.064 & 0.683 & -0.007 & 0.962 \\
FACT-F & -0.129 & 0.411 & 0.040 & 0.797 & -0.137 & 0.382 & -0.026 & 0.868 \\
\hline
\end{tabular}

LBM: Lean Body mass; CRP: C-reactive protein; EPA: eicosapentanoic acid; DHA: docosahexanoic acid; FACT-B + 4: Quality of life with breast related items; FACT-F: Fatigue. 
Table 4 Best predictors of LBM post-treatment using hierarchical regression

\begin{tabular}{|c|c|c|c|}
\hline Predictor & $\begin{array}{c}\text { Regression coefficient* } \\
(95 \% \mathrm{Cl})\end{array}$ & p-value** & Adjusted $\mathrm{R}^{2 \mathrm{t}}$ \\
\hline Weight & 0.948 & 0.000 & 0.634 \\
\hline $\begin{array}{l}\text { Stage Tmill } \\
\text { completed }\end{array}$ & 0.225 & 0.007 & 0.713 \\
\hline $\begin{array}{l}\text { No. push } \\
\text { ups (1 } \mathrm{min})\end{array}$ & 0.275 & 0.002 & 0.764 \\
\hline
\end{tabular}

*Regression coefficients taken from final model including: body mass, number push-ups performed, treadmill stage reached.

**Significance of the individual predictor within the final model.

tDenotes value reported as each variable was added into the model in the order: body mass, stage tmill completed then no. push ups

$\mathrm{Cl}=$ confidence interval.

the $\%$ time spent in $\geq$ moderate intensity physical activity, stage achieved on sub-maximal treadmill test and number of push-ups completed. To the authors' knowledge, this is the first study to determine associations between physical function and body composition in women who have completed treatment for breast cancer.

Our results agree with previous cross-sectional and prospective cohort studies, which have shown that decreasing physical activity levels are associated with greater adverse body composition change,(Irwin et al. 2003; Irwin et al. 2005) while dietary measures(DemarkWahnefried et al. 2001) have been less predictive of these changes. The findings relating to the influence of chemotherapy on LBM agree with two previous studies (Campbell et al. 2007; Winters-Stone et al. 2009) but are in contrast to five studies that have shown a greater decrease in LBM after chemotherapy (Cheney et al. 1994; Demark-Wahnefried et al. 1997; Demark-Wahnefried et al. 2001; Gordon et al. 2011; Kutynec et al. 1999). In addition, Prado et al. reported that individuals with chemotherapy toxicity had a greater risk of sarcopenia (Prado et al. 2009). Differences in our results may be due to the cross-sectional nature of the study. Previously published data sets indicating LBM change after chemotherapy and hormonal therapies were prospective in nature (Cheney et al. 1994; Demark-Wahnefried et al. 1997; Demark-Wahnefried et al. 2001; Gordon et al. 2011; Kutynec et al. 1999) and were able to see trends over time.
No associations were found between erythrocyte LCn-3 and markers of body composition. Recent studies in populations during and post-chemotherapy treatment have indicated a positive relationship between skeletal muscle mass and plasma phospholipid LCn-3 content (Murphy et al. 2010; Murphy et al. 2011), however these participants experienced significant and rapid muscle loss during treatment. After early stage breast cancer treatment, the rate and magnitude of muscle loss experienced is not typically as high as when compared to more advanced staged cancers (Mcdonald et al. 2011; Murphy et al. 2010). As a result, our results are comparable to metabolic/obese populations undergoing similar body composition change (Krebs et al. 2006; Noreen et al. 2010; Storlien et al. 2001).

Total body mass, push-ups performed in one-minute, and stage completed on treadmill remained in the final model accounting for $76 \%$ of the variation in LBM. These results are of interest as they indicate an association with physical function and healthier body composition. Specifically, the strength of association with number of push-ups/minute as opposed to squats may indicate the importance of whole body resistance training to maintain or achieve a higher LBM and lower fat mass. A decrease in sports/recreational exercise has been previously associated with an increase in adiposity however, LBM change was not reported (Irwin et al. 2005). It is possible that those who performed more push-ups due to an increase in relevant exercise training may also be more conscientious in regards to dietary intake, however no association was found in this study.

Both erythrocyte DHA and EPA content were associated with markers of physical function, surprisingly in positive and negative directions, respectively. DHA was strongly and independently associated with the ability to perform push-ups, while erythrocyte EPA content was negatively associated with squats performed. In addition, assessing predictive models for push-up performance, when\%time $\geq$ moderate physical activity was added to the DHA model, a greater effect was seen. In contrast, EPA content remained significantly negatively associated with squats performed. Previous studies have indicated an increase in muscle protein synthesis (Smith et al. 2011a;

Table 5 Correlations between measures of physical function and LCn-3 content of erythrocytes

\begin{tabular}{|c|c|c|c|c|c|c|c|c|c|c|}
\hline \multirow[t]{2}{*}{ Physical function* } & \multicolumn{2}{|c|}{$\%$ time $>$ mod } & \multicolumn{2}{|c|}{ EPA } & \multicolumn{2}{|c|}{ EPA adj** } & \multicolumn{2}{|c|}{ DHA } & \multicolumn{2}{|c|}{ DHA adj.** } \\
\hline & $r$ & $p$-value & $r$ & p-value & $\beta$ & p-value & $r$ & pcs-value & $\beta$ & $\mathrm{p}$-value \\
\hline Push ups & 0.467 & 0.001 & 0.072 & 0.648 & 0.212 & 0.118 & 0.517 & 0.000 & 0.399 & 0.001 \\
\hline Squats & 0.110 & 0.479 & -0.338 & 0.029 & -0.268 & 0.041 & 0.153 & 0.333 & -0.37 & 0.776 \\
\hline Handgrip & -0.068 & 0.663 & 0.083 & 0.603 & -0.144 & 0.340 & 0.109 & 0.492 & 0.099 & 0.482 \\
\hline Treadmill & 0.224 & 0.138 & -0.11 & 0.493 & 0.13 & 0.929 & 0.267 & 0.083 & 0.147 & 0.277 \\
\hline
\end{tabular}

*Push ups: performed in one-minute; Squats: performed in one-minute; Stage of treadmill completed: at which $85 \%$ of estimated HRmax was reached.

$\%$ time > mod: moderate \& vigorous data combined; EPA: eicosapentanoic acid; DHA: docosahexanoic acid.

**Fully adjusted model included: weight, age, \% time > mod activity \& LBM. Correlation coefficient ( $\beta$ ) 
Smith et al. 2011b) and peak torque development (Rodacki et al. 2012) after supplementation of LCn-3 s was combined with an anabolic stimulus. In advanced cancer populations, EPA LCn-3 supplementation (often in conjunction with a protein-rich supplement) has been associated with improvements in physical function (Moses et al. 2004) and strength (Fearon et al. 2006), while EPA and DHA LCn-3 + NSAIDs have been shown to improve handgrip strength (Cerchietti et al. 2007). Our results both agree and disagree with the previous literature base with no clear reason for the opposing directions for the associations between physical function, DHA and EPA. Further investigation into LCn-3 and physical activity interactions are required.

Our population compared favourably with larger cohorts for body composition, (Chlebowski et al. 2002; Irwin et al. 2005) education level, (Irwin et al. 2005) however the exclusion of those with a diagnosed chronic disease (T2DM or CVD) and those who could not participate in moderate physical activity, may have led to our participants being younger and more physically active than the general breast cancer population.

\section{Conclusion}

This is the first study to report that higher weight adjusted LBM is associated with greater upper body strengthendurance and aerobic fitness in women after completion of treatment for breast cancer. Further research is required to elucidate LCn-3-exercise interactions.

\section{Competing interest}

All capsules for the intervention were provided by Blackmores Pty Ltd; no intellectual input regarding study design, data collection, analysis or write up was given.

\section{Authors' contribution}

CM-Contributed to study design, carried out intervention phase, data collection \& analysis, write up; JB-Study design, input into analysis and interpretation, intellectual input for write up; SC- study design, analysis and interpretation, write up; MW- Statistician, analysis and write up. All authors read and approve the final manuscript.

\section{Acknowledgements}

Funding for the blood analyses, exercise equipment other costs were provided by a grant from the Wesley Research Institute (Grant no. 1034).

Received: 5 November 2013 Accepted: 8 November 2013

Published: 19 December 2013

\section{References}

American College of Sports Medicine (2010) ACSM's Guidelines for Exercise Testing and Prescription, 8th edn. Wolters Kluwer/Lippincott Williams \& Wilkins, Philadelphia

Campbell KL, Lane K, Martin AD et al (2007) Resting Energy Expenditure and Body Mass Changes in Women During Adjuvant Chemotherapy for Breast Cancer. Cancer Nursing 30:95-100

Cella D, Tulsky D, Gray G et al (1993) The Functional Assessment of Cancer Therapy scale: Development and validation of the general measure. J Clin Oncol 11:570-579

Cerchietti LCA, Naviganteac AH, Castroa MA (2007) Effects of Eicosapentaenoic and Docosahexaenoic n-3 Fatty Acids From Fish Oil and Preferential Cox-2 Inhibition on Systemic Syndromes in Patients With Advanced Lung Cancer. Nutr Cancer 59:14-20
Cheney CL, Mahloch J, Freeny P (1994) Computerised tomography assessment of women with weight changes associated with adjuvant treatment for breast cancer. Am J Clin Nutr 66:141-146

Chlebowski RT, Aiello E, Mctiernan A (2002) Weight Loss in Breast Cancer Patient Management. J Clin Oncol 20:1128-1143

Courneya KS, Segal RJ, Mackey JR et al (2007) Effects of Aerobic and Resistance Exercise in Breast Cancer Patients Receiving Adjuvant Chemotherapy: A Multicenter Randomized Controlled Trial. J Clin Oncol 25:4396-4404

Demark-Wahnefried W, Hars V, Conaway MR et al (1997) Reduced rates of metabolism and decreased physical activity in breast cancer patients receiving adjuvant chemotherapy. Am J Clin Nutr 65:1495-1501

Demark-Wahnefried W, Peterson BL, Winer EP et al (2001) Changes in Weight, Body Composition, and Factors Influencing Energy Balance Among Premenopausal Breast Cancer Patients Receiving Adjuvant Chemotherapy. Journal of Clinical Oncology 19:2381-2389

Dewey A, Baughan C, Dean T, Higgins B, Johnson I (2007) Eicosapentaenoic acid (EPA, an omega-3 fatty acid from fish oils) for the treatment of cancer cachexia. The Cochrane database of systematic reviews (1469-493X) 1:CD004597

Fearon KCH, Barber MD, Moses AG et al (2006) Double-Blind, Placebo-Controlled, Randomized Study of Eicosapentaenoic Acid Diester in Patients With Cancer Cachexia. Journal of Clinical Oncology 24:3401-3407

Fearon KCH, Von Meyenfeldt MF, Moses AGW et al (2003) Effect of a protein and energy dense $\mathrm{n}-3$ fatty acid enriched oral supplement on loss of weight and lean tissue in cancer cachexia: a randomised double blind trial. Gut 52:1479-1486

Francini G, Petrioli R, Montagnani A et al (2006) Exemestane after tamoxifen as adjuvant hormonal therapy in postmenopausal women with breast cancer: effects on body composition and lipids. Br J Cancer 95:153-158

Gordon AM, Hurwitz S, Shapiro CL et al (2011) Premature ovarian failure and body composition changes with adjuvant chemotherapy for breast cancer. Menopause 18:1244

Healy LA, Ryan AM, Carroll P et al (2010) Metabolic Syndrome, Central Obesity and Insulin Resistance are Associated with Adverse Pathological Features in Postmenopausal Breast. Clinical Oncology 22:281-288

Ibrahim EM, Al-Homaidh A (2011) Physical activity and survival after breast cancer diagnosis: meta-analysis of published studies. 28(3):65-753. doi:10.1007/ s12032-010-9536-x. Epub 2010 Apr 22

Irwin ML, Alvarez-Reeves M, Cadmus L et al (2009) Exercise Improves Body Fat, Lean Mass, and Bone Mass in Breast Cancer Survivors. Obesity 17:1534-1541

Irwin ML, Crumley D, Mctiernan A et al (2003) Physical activity levels before and after a diagnosis of breast carcinoma. Cancer 97:1746-1757

Irwin ML, Mctiernan A, Baumgartner RN et al (2005) Changes in body fat and weight after a breast cancer diagnosis: influence of demographic, prognostic, and lifestyle factors. Journal of Clinical Oncology 23:774-782

Krebs JD, Browning LM, Mclean NK et al (2006) Additive benefits of long-chain $\mathrm{n}-3$ polyunsaturated fatty acids and weight-loss in the management of cardiovascular disease risk in overweight hyperinsulinaemic women. Int $J$ Obes 30:1535-1544

Kutynec Cl, Mccargar L, Barr Sl et al (1999) Energy balance in women with breast cancer during adjuvant treatment. Journal of the American Dietetic Association 99:1222-1227

Martin GS (2004) The interviewer-administered, open-ended diet history method for assessing usual dietary intakes in clinical research: relative and criterion validation studies. In: University of Wollongong Thesis Collection. University of Wollongong, Wollongong

Mcdonald C, Bauer JM, Capra S et al (2013a) The Muscle mass, Omega-3, Diet, Exercise and Lifestyle (MODEL) study - study protocol for a randomised controlled trial for women who have completed breast cancer treatment. BMC Cancer, In Press

Mcdonald CK, Bauer JD, Capra S (2011) Body Composition and Breast Cancer- the Role of Lean Body Mass. Cancer Forum 35:102-106

Mcdonald CK, Bauer JD, Capra S (2013b) Omega-3 fatty acids and changes in LBM - alone or in synergy for better muscle health? A Review. Canadian Journal of Physiology and Pharmacology, in press

Mcneely ML, Campbell KL, Rowe BH et al (2006) Effects of exercise on breast cancer patients and survivors: a systematic review and meta-analysis. CMAJ: Canadian Medical Association Journal 175:34-41

Montagnani A, Gonnelli S, Cadirni A et al (2008) The effects on lipid serum levels of a 2-year adjuvant treatment with exemestane after tamoxifen in postmenopausal women with early breast cancer. European Journal of Internal Medicine 19:592-597 
Moses AW, Slater C, Preston T et al (2004) Reduced total energy expenditure and physical activity in cachectic patients with pancreatic cancer can be modulated by an energy and protein dense oral supplement enriched with n-3 fatty acids. Br J Cancer 90:996-1002

Mourtzakis M, Bedbrook M (2009) Muscle atrophy in cancer: a role for nutrition and exercise. Applied Physiology, Nutrition and Metabolism 34:950-956

Murphy RA, Mourtzakis M, Chu QS et al (2010) Skeletal Muscle Depletion Is Associated with Reduced Plasma (n-3) Fatty Acids in Non-Small Cell Lung Cancer Patients. The Journal of Nutrition 140:1602-1606

Murphy RA, Mourtzakis M, Chu QSC et al (2011) Nutritional intervention with fish oil provides a benefit over standard of care for weight and skeletal muscle mass in patients with nonsmall cell lung cancer receiving chemotherapy. Cancer 117:1775-1782

Murphy RA, Mourtzakis M, Mazurak VC (2012) n-3 polyunsaturated fatty acids: the potential role for supplementation in cancer. Current opinion in clinical nutrition and metabolic care 15:246

Newman AB, Kupelian V, Visser M et al (2006) Strength, But Not Muscle Mass, Is Associated With Mortality in the Health, Aging and Body Composition Study Cohort. The Journals of Gerontology Series A: Biological Sciences and Medical Sciences 61:72-77

Noreen Eric E, Sass Michael J, Crowe Megan L, Pabon Vanessa A, Brandauer J, Averill Lindsay K (2010) Effects of supplemental fish oil on resting metabolic rate, body composition, and salivary cortisol in healthy adults. Journal of the International Society of Sports Nutrition, ISSN 1550-2783 7(1):31

Pedersen BK, Febbraio MA (2012) Muscles, exercise and obesity: skeletal muscle as a secretory organ. Nature reviews Endocrinology 8:457

Pierce BL, Ballard-Barbash R, Bernstein L et al (2009) Elevated Biomarkers of Inflammation Are Associated With Reduced Survival Among Breast Cancer Patients. J Clin Oncol 27:3437-3444

Prado CMM, Baracos VE, Mccargar LJ et al (2009) Sarcopenia as a Determinant of Chemotherapy Toxicity and Time to Tumor Progression in Metastatic Breast Cancer Patients Receiving Capecitabine Treatment. Clinical Cancer Research 15:2920-2926

Ries A, Trottenberg P, Elsner F et al (2012) A systematic review on the role of fish oil for the treatment of cachexia in advanced cancer: an EPCRC cachexia guidelines project. Palliat Med 26:294-304

Rodacki CL, Rodacki AL, Pereira G et al (2012) Fish-oil supplementation enhances the effects of strength training in elderly women. The American Journal of Clinical Nutrition 95:428-436

Ruiz JR, Sui X, Lobelo F et al (2008) Association between muscular strength and mortality in men: prospective cohort study. British Medical Journal (Clinical Research Ed) 337:a439-495

Schmitz KH, Ahmed RL, Hannan PJ et al (2005) Safety and Efficacy of Weight Training in Recent Breast Cancer Survivors to Alter Body Composition, Insulin, and Insulin-Like Growth Factor Axis Proteins. Cancer Epidemiology, Biomarkers \& Prevention 14:1672-1680

Sharman A, Stowasserb M (2009) Australian Association for Exercise and Sports Science Position Statement on Exercise and Hypertension. Journal of Science and Medicine in Sport 12:252-257

Sheean PM, Hoskins K, Stolley M (2012) Body composition changes in females treated for breast cancer: a review of the evidence. Breast Cancer Res Treat 135:663-680

Smith Gl, Atherton P, Reeds DN et al (2011a) Dietary omega-3 fatty acid supplementation increases the rate of muscle protein synthesis in older adults: a randomized controlled trial. Am J Clin Nutr 93:402-412

Smith Gl, Atherton P, Reeds DN et al (2011b) Omega-3 polyunsaturated fatty acids augment the muscle protein anabolic response to hyperinsulinaemiahyperaminoacidaemia in healthy young and middle-aged men and women Clinical science (London, England: 1979) 121:267-278

Storlien LH, Robertson RM, Hill JO et al (2001) Role of weight loss and polyunsaturated fatty acids in improving metabolic fitness in moderately obese, moderately hypertensive subjects. J Hypertens 19:1745-1754

Van Der Meij BS, Van Bokhorst-De Van Der Schueren MA, Langius JA et al (2011) $n-3$ PUFAs in cancer, surgery, and critical care: a systematic review on clinical effects, incorporation, and washout of oral or enteral compared with parenteral supplementation. The American Journal of Clinical Nutrition 94:1248-1265
Van Londen G, Perera S, Vujevich K et al (2011) The impact of an aromatase inhibitor on body composition and gonadal hormone levels in women with breast cancer. Breast Cancer Res Treat 125:441-446

Winters-Stone KM, Nail L, Bennett JA et al (2009) Bone health and falls: fracture risk in breast cancer survivors with chemotherapy-induced amenorrhea. Oncology Nursing Forum 36:315-325

doi:10.1186/2193-1801-2-681

Cite this article as: McDonald et al:: Muscle function and omega-3 fatty acids in the prediction of lean body mass after breast cancer treatment. SpringerPlus 2013 2:681.

\section{Submit your manuscript to a SpringerOpen ${ }^{\odot}$ journal and benefit from:}

- Convenient online submission

- Rigorous peer review

- Immediate publication on acceptance

- Open access: articles freely available online

- High visibility within the field

- Retaining the copyright to your article

Submit your next manuscript at $>$ springeropen.com 